\title{
Automatic Game Experience Identification in Educational Games
}

\author{
Wilk Oliveira $^{1}$, Luiz Rodrigues ${ }^{1}$, Armando M. Toda ${ }^{1}$, Paula T. Palomino ${ }^{1}$, \\ Seiji Isotani ${ }^{1}$ \\ ${ }^{1}$ Institute of Mathematical and Computer Sciences - University of São Paulo (USP) \\ \{wilk.oliveira, lalrodrigues, armando.toda, paulatpalomino\}@usp.br, \\ sisotanieicmc.usp.br
}

\begin{abstract}
One of the main challenges in the field of educational games is the automatic and implicit users' game experience identification. To face this challenge, we present an exploratory study by using a data-driven based approach for collecting and identifying this experience. We used two different data-mining techniques aiming to associate the user's data logs from an educational game with their game-like experience. Our main results indicate that it is possible to extract the automatic and implicit acquisition of the student's game experience in educational games and demonstrate how user's data logs drive their experiences. We also provided different associations between user data logs in educational games and the student's game experience.
\end{abstract}

\section{Introduction}

One of the most important challenges on game design (specially when designing educational games) is to identify the student's game experience in order to evaluate the game and provide a better design solution for the gamer [Petri and von Wangenheim 2016]. According to [Petri and von Wangenheim 2016], currently, this evaluation is conducted using questionnaires and/or surveys by asking the respondents about their experience in the game. Although it is widely used approach, it is considered intrusive and directly influence the player experience, affecting their immersion in the game. This also may cause stress or other kind of discomfort, leading they to respond differently from their actual experience [Spector 1994]. Another issue is the complexity of data analysis and the fact that in some cases is unfeasible to apply some types of questionnaires on a large scale (with a large number of users) [Wood et al. 2004].

Thus, a possible and emerging solution is the automatic identification and prediction of the players' experience (i.e., player modelling) based on the system data logs, which are generated during their interactions within the system [Hooshyar et al. 2017, Oliveira et al. 2019]. In other words, the idea of taking advantage of user data logs in the game and use artificial intelligence (AI) techniques to uncover behavior patterns associated with the players experience as well as predict the players' experience based on their behavior (data log) [Charleer et al. 2018, Santos and Isotani 2018].

Based on the exposed, this study explore a data-driven based approach to associate the students' data log in an educational game with their experience in the game obtained through a game user experience survey. We conducted two studies using two different data mining techniques. The main results indicate that it is possible to relate different 
VIII Congresso Brasileiro de Informática na Educação (CBIE 2019)

Anais do XXX Simpósio Brasileiro de Informática na Educação (SBIE 2019)

students' data log with their experience in the game. Following, this paper presents a background of game experience, player modeling, as well as related works in the Section 2. Then, the Section 3 describes the study's experiment, followed by the Section 4 that presents and discusses the experiment's findings. Thereafter, the Sections 5 discusses threats to the experiment validity and the Section 6 draws concluding remarks.

\section{Background}

\subsection{Game Experience}

Game experience is a complex aspect that has been analyzed through different methods [Zammitto 2009]. In addition to the natural difficult of measuring players' psychological concepts, evaluating game experience is hard because player experience is often related to various factors [Mekler et al. 2014]. Especially in educational games, researchers usually concern education-related aspects, such as learning gains or flow state (e.g., [Rodrigues et al. 2017, Santos et al. 2017]), not focusing on the game experience itself [Eckardt and Robra-Bissantz 2018]. This is harmful to the student, since the technology is unable to provide positive experiences, players are unlikely to interact with it [Bauckhage et al. 2012]. Thereby, the importance of delivering both educational and general purpose games that provide players with positive game experiences.

\subsection{Player Modelling}

Research on computational models of players in a game (i.e. player modeling), aims at understanding how the player-game interaction is experienced [Yannakakis et al. 2013]. This understanding might be achieved through theory or data-driven approaches, wherein the former mostly relies on specialist knowledge for model development and the latter is mostly based on game data and players feedback to derive models [Yannakakis and Togelius 2011]. Despite theory-driven methods have shown their worth, relying on the developer's knowledge limits its effectiveness, whilst data-driven approaches are promising techniques which have the potential to tackle that limitation of the former [Hooshyar et al. 2018].

Furthermore, data-driven player modeling methods have shown benefits for educational games, as discussed in [Hooshyar et al. 2017], which found that (i) most studies modeled players in-game behavior and (ii) the most used techniques are supervised and unsupervised learning. For games in general, the findings were similar [Hooshyar et al. 2018].

\subsection{Related Works}

Given the relevance of providing positive experiences for players, studies have explored player modeling as a means to automatically identify their game experience at playing time. In their seminal paper, [Pedersen et al. 2010] used players' self-reports to model their experiences in terms of fun, challenge, frustration, predictability, anxiety, and boredom. Following that study, many other were conducted in a similar fashion, such as [Shaker et al. 2010] and [Xu et al. 2016], which modeled players' fun, challenge, and frustration in two general-purpose games. Despite the valuable contribution of those studies, three limitations must be highlighted. First, the studies measure player experience via preference, e.g. version A was more fun than version B. Consequently, their player model 
is unable to indicate the extent of the experience (e.g., moderate or fair). Second, the developed models do not consider that game changes will affect players' experience. Third, players' experience is captured after interacting with few levels (e.g., four), which might be problematic because of the novelty effect.

This study tackles the aforementioned limitations by $(i)$ measuring players' experience via ratings, which allows the model to predict the extent of players' game experience, (ii) relying on log data which considers players' behavior over time, which will change the model's outputs accordingly to their in-game behavior, and (iii) capturing players' feedback after 30 minutes of gameplay. Furthermore, most data-driven player modeling approaches applied in educational games focused on behavior and movement rather than their experiences (e.g., emotional affects/aspects) [Hooshyar et al. 2017]. Unlike this trend, [Lara et al. 2018] developed a model able to predict players' experience in terms of emotions (e.g., happy, frustrated, excited, bored). However, their approach requires players to speak in order to predict their experiences. In this study, only data log are used as the model's input, which that requirement (speaking). Additionally, this study relies on interpretable algorithms for model development, providing insights into what/how players' in-game behavior drive their experiences, which is another contribution compared to the other studies.

\section{Method}

Our study is characterized as an exploratory study using a data-driving approach, comparing different student's data logs generated during the use of an educational game, with the experience of the players in the game.

\subsection{Participants}

Initially, our participants were 178 Brazilian elementary school students and after the data treatment, we conducted our study with 162 students (94 male and 28 female), with a mean age of 14 years. Thus, the study is in according with different criteria for an adequate sample size. According to [Bentler and Chou 1987] there must be a minimum ratio of 5 respondents per 1 construct in the model and [Loehlin 1998] suggests that at least 100 participants are required for a complete sample size.

\subsection{Materials}

In order to obtain the student's data logs with their game experience, we used the the game SpaceMath [Rodrigues and Brancher 2018, Rodrigues and Brancher 2019]. It was chosen by convenience because access to in game data log were granted by the game developer. The game aims to help students to practice basic Mathematical equations (addition, subtraction, multiplication, and division) by repeating mathematical operations. After starting the game, the student can move their avatar through the space and teleport blocks that hide the numbers that solve an equation that appears at the top of the screen. The student must identify the numbers in the correct order to trigger the next phase, as presented in the Figure 1.

To obtain the students data logs, it was implemented in the game a module to get different students' interaction. The module was capable to obtain the following data: mean_playerwon $=$ the number of level won divided by the number of 
VIII Congresso Brasileiro de Informática na Educação (CBIE 2019)

Anais do XXX Simpósio Brasileiro de Informática na Educação (SBIE 2019)

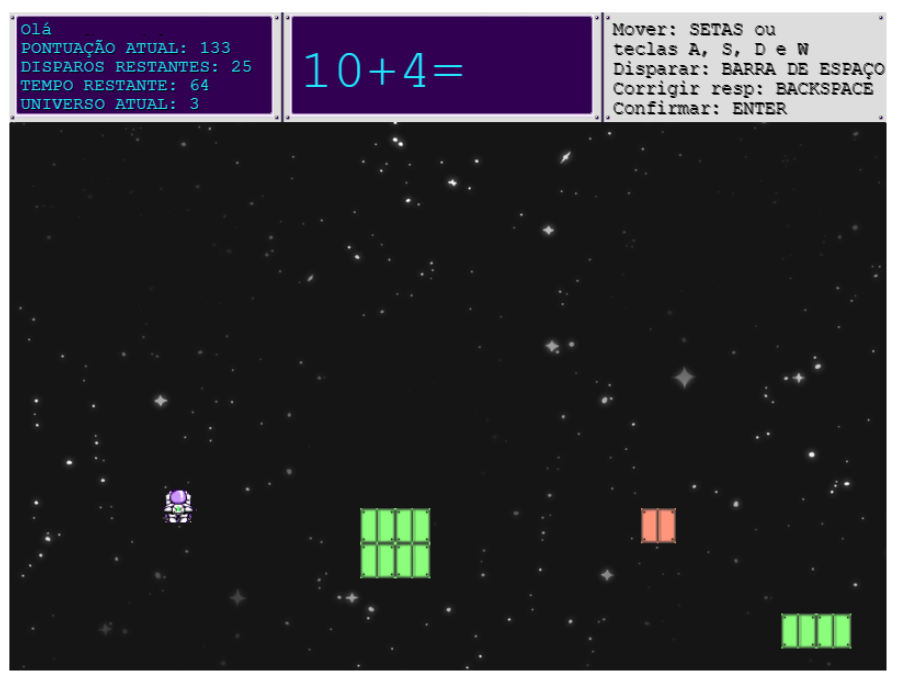

Figure 1. SpaceMath Game Interface

played levels; mean_timespent $=$ the average time (seconds) spend per level; mean_score $=$ the average score per level; mean_summedscore $=$ the average summed score per level; max_summedscore $=$ the highest score achieved; mean_winsstreak $=$ the average win-streak; max_winsstreak = the highest win-streak achieved; max_totalplayed $=$ the total levels played; mean_shotsfired $=$ the average of shots fired per level; mean_enemiesTeleported $=$ the average of enemies (aliens) teleported per level; mean_boxesTeleported $=$ the average of boxes teleported per level; mean_shotsAvailable $=$ the average of shots available per level; sd_player = the standard deviation of the win/loss ratio; med_player $=$ the median of the win/loss ratio; var player $=$ the variance of the win/loss ratio.

To collect the students' game experience, we used "The Game Experience Questionnaire (GEQ)" proposed and validated by [IJsselsteijn et al. 2013]. The GEQ is composed of 14 non-invasive questions related to the user game experience (e.g., "I forgot everything around me") organized in a five-point Likert-Scale [Likert 1932] (not at all, slightly, moderately, fairly, or extremely). The original questionnaire was made in English, so we validated it to Brazilian Portuguese to use it in our context. The validation followed the one proposed in [Borges et al. 2017], where: (a) we translated the text and verified through specialists and native speakers; $(b)$ then we applied the Cronbach's alpha test for reliability and consistency 1 , $c$ ) finally, we measured the adequacy of our sampling and applied the Bartlett's test to verify the homogeneity of our variances.

The results indicated that the used questionnaire has a reasonable internal consistence (Cronbach's $\alpha=0,759$ ) and using the Kaiser-Meyer-Olkin (KMO) [Dziuban and Shirkey 1974] test to measure our sample adequacy we identified that our data was adequate with a KMO $=0.830$. Our Bartlett's test scored a K-squared of 70,629 ( $p$-value $=6,15 \mathrm{e}-10)$ with a $\mathrm{p}$-value greater than 0.05 . Thus, the questionnaire used in this experiment may be considered adequate for the studies presented in this paper, as well as for the conduction of new studies. The complete analysis (including the used scrips) can found in the following link: http://twixar.me/f511

\footnotetext{
${ }^{1}$ Analysis were made using $\mathrm{R}$ and psych package
} 
VIII Congresso Brasileiro de Informática na Educação (CBIE 2019)

Anais do XXX Simpósio Brasileiro de Informática na Educação (SBIE 2019)

\subsection{Procedure}

The study procedure was organized in two general steps: ( $i$ ) data processing; and (ii) data mining. In the first step, we removed data logs from the students that do not answer the GEQ or with incomplete answers. We also removed data from students who played the game for less than 30 minutes (remaining with data of 162 students). We also calculated the "five number summary" (the minimum value, the first quartile, the median, the third quartile, and the maximum value of a set of numbers (data)) [Hoaglin et al. 2000] and the mean, median, variance, and standard deviation of each available variable.

In the second step, we performed two different data mining techniques in order to relate the log data provided by the game to the students' experience provided by the GEQ. First, we used decision tree (DT), that is a non-parametric supervised learning method used for classification and regression, because it yields interpretable classifiers (i.e., why a player's game experience was fair) [Huang et al. 2011]. We used the algorithm J48 that is an is an open source Java implementation of the C4.5 algorithm [Kaur and Chhabra 2014], which is one of the most widely used decision trees algorithm, because its capacity to perform well in terms of accuracy regardless of the dataset [Kaur 2018]. Second, we used Association Rule Mining (ARM) to identify if-then additional patterns, if not found by DT, and to check whether those corroborate. Here, we transformed the numerical data referring to the data log obtained into nominal values based on its "five number summary" and used the criteria support and confidence to identify the most important relationships. We used the Apriori algorithm that is one of the most popular to find frequent itemsets from a transaction data set and derive association rules [Wu et al. 2008].

\section{Results and Discussions}

This sections presents and discusses this paper's experiment's results. First, the section introduces the statistical distribution of the log data that collected. Thereafter, the results of modeling players' game experience through both decision trees and association rules are shown. Table 1 demonstrates the five number summary of the log data from players included in this study's experiment. It shows the minimum, first quartile, median, third quartile, and the maximum value for each of log data type.

First, based on the data log, we constructed a Decision Tree (Figure 2$)$ to predict whether the players' game experience was not at all, slight, moderate, fair, or extremely. As shown in Figure 2, only four out of the 15 attributes were relevant for the tree. The four selected attributes are related to the player's performance, which represents the players' median and SD of win/loss ratio, and the average of both score per level as well as summed score per levels. Hence, in order to predict a player's game experience, given an input set (log data), one must read the decision tree in a top-down fashion, comparing each attribute (e.g., med_player) to the threshold presented below each node to determine whether the flow should go to the right or to the left. This process must be repeated until reaching a leaf node, which indicates the game experience. For instance, a player with median and SD of win/loss ratio of 5 and 1, respectively, would go down to the left most leaf, which indicates this player's game experience was fair.

In terms of performance, the decision tree was validated through a 10-folds crossvalidation process, which yielded an average accuracy of 0.796 and a average kappa 
VIII Congresso Brasileiro de Informática na Educação (CBIE 2019)

Anais do XXX Simpósio Brasileiro de Informática na Educação (SBIE 2019)

Table 1. Five number summary

\begin{tabular}{l|l|l|l|l|l}
\hline & min & 1st qrt & med & 3rd qrt & max \\
\hline mean_playerwon & 000.762 & 000.865 & 000.886 & 000.915 & 0000.980 \\
mean_timespent & 012.288 & 022.392 & 027.160 & 031.368 & 0052.059 \\
mean_score & 041.647 & 053.162 & 054.885 & 056.799 & 0061.793 \\
mean_summedscore & 111.765 & 218.521 & 287.289 & 330.744 & 0756.857 \\
max_summedscore & 268.000 & 591.500 & 748.352 & 855.250 & 1539.000 \\
mean_winsstreak & 001.932 & 003.384 & 004.524 & 005.288 & 0012.571 \\
max_winsstreak & 005.000 & 010.000 & 012.537 & 015.000 & 0028.000 \\
max_totalplayed & 033.000 & 056.000 & 068.821 & 078.000 & 0145.000 \\
mean_shotsfired & 003.700 & 007.928 & 010.137 & 011.174 & 0029.347 \\
mean_enemiesTeleported & 000.016 & 000.560 & 001.258 & 001.608 & 0006.878 \\
mean_boxesTeleported & 002.017 & 005.630 & 007.687 & 009.279 & 0021.449 \\
mean_shotsAvailable & 009.849 & 019.243 & 024.456 & 028.327 & 0053.119 \\
sd_player & 000.000 & 001.041 & 001.264 & 001.517 & 0001.940 \\
med_player & 001.000 & 002.500 & 003.160 & 004.000 & 0005.000 \\
var_player & 000.000 & 001.084 & 001.731 & 002.302 & 0003.764 \\
\hline Key: & & & & &
\end{tabular}

Key: min: minimum value; 1 st qrt: first quartile; med: median:

3rd qrt: third quartile; max: maximum value.

coefficient $(\kappa)$ of 0.649 . Therefore, considering recommendations for $\kappa$ interpretation [Viera et al. 2005], we can conclude that the developed tree achieved a fair agreement ( $\kappa$ between 0.6 and 0.8 ) to the ground truth, based on the validation process. Thus, the contribution of those findings are twofold. One is that the decision three might be used to assess game experience in real time, providing a fair performance when predicting the extent of players' experience, and has the potential to aid on the process of game adaptation/automatic content generation. The other is that the decision three's structure allows the interpretability of which game factors influence game experience, as well as it demonstrates how each of those affect players (e.g., large variation on win/loss ratio is determinant in whether player' experience will be extreme or fair).

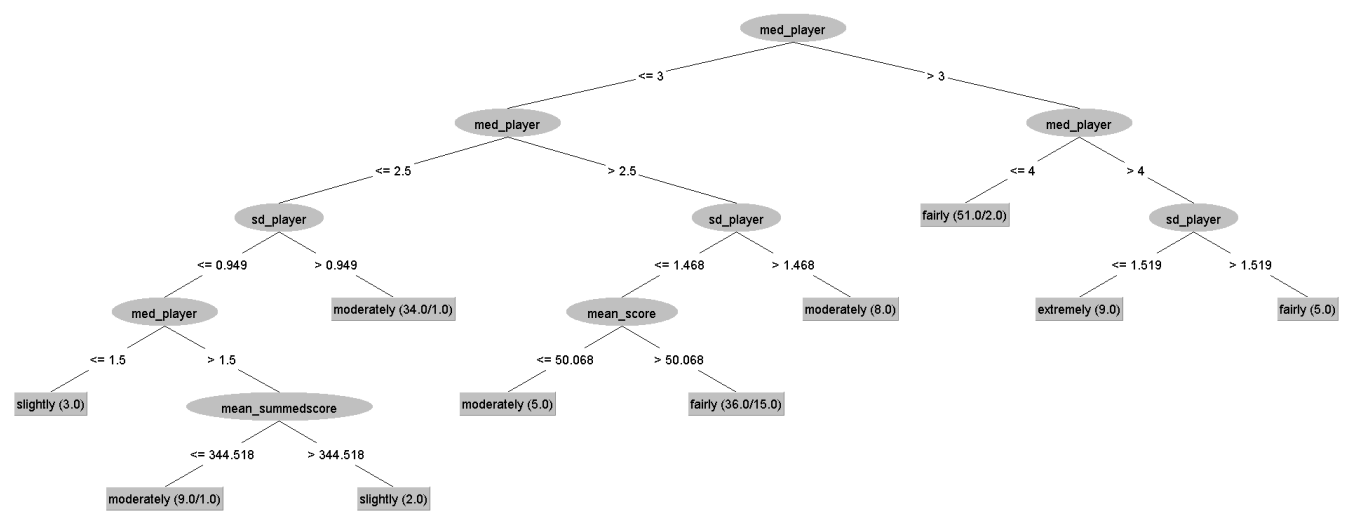

Figure 2. Decision Tree

Second, for the association from data log to game experience, we used only rules with confidence level greater than or equal to $90 \%$. Although the algorithm was capable 
VIII Congresso Brasileiro de Informática na Educação (CBIE 2019)

Anais do XXX Simpósio Brasileiro de Informática na Educação (SBIE 2019)

of identify $\left.2011\right|^{2}$ different rules, only three of these were related to the game experience class, i.e. game experience level such as extremely or fairly is at after the then part of the rules. This shows that there are multiple data log that are related to each other, despite few of these data are related to game experience with a high degree of confidence. This corroborates with the decision tree previously found, which reduced the initial 15 attributes to just 4 . Nevertheless, the three rules which related to some extent of game experience are following described.

- If $s d$ player $=$ very $h i g h$ and med_player $=$ very high then game experience $=$ fairly (confidence: 1; lift: 2.13; lev: 0.07): This rule is supported by the fact that all 21 players with very high SD and median of win/loss ratio had fairly game experiences. That is, those that win way more than lose but with a very high deviance reported fair game experiences.

- If med player $=$ very high and var_player $=$ very_high 21 then game experience = fairly (confidence: 1; lift: 2.13; lev: 0.07): This rule is supported by the fact that the 21 players with a very high median and variance of win/loss ratio had fairly game experiences. That is, those that win way more than lose but with a highly variant performance in this aspect reported fairly game experiences.

- If sd_player $=$ very high and med player $=$ very_high and var player $=$ very high then game experience = fairly (confidence: 1; lift: 2.13; lev: 0.07): This rule reflects the other two in conjunction, demonstrating that players with very high $\mathrm{SD}$, variance, and median of win/loss ratio reported fairly experiences.

Analyzing the association rules reinforced the indication that players' performance was the key factor to determine their reported game experiences. Furthermore, only those rules in which a game experience class was at the then side was presented above. However, there are other rules that involve these classes at the if side, with a confidence level of at least $90 \%$ as well. For instance, those rules suggested that players who reported moderated game experiences teleporting very little boxes to outside the level and played few levels. Players that teleport few boxes might be those that are conservative, teleporting just the necessary amount of boxes, at the same time might be those who faced difficulties to play. This is in line with those who played few levels and, probably, did not take the the time necessary to learn the game and to start to yield good performances.

Moreover, those who had poor performances (low and very low) in terms of, e.g. win/loss ratio, average score per level, summed score per level, and the highest winstreak achieved, also reported moderate experiences. This suggests the hypothesis that facing difficulties while playing, on the basis of the aforementioned metrics, might be a factor that leads players to report moderate game experiences. Thus, the association rules analysis provided insights into possible in-game behaviors that can lead players to (just) experience moderated game experiences. Thereby, these concerns are of value to designers/developers to take into account in their games' development or in the design of game adaptation mechanisms based on player behavior.

\section{Threats to Validity}

For this study, limitations such as the sample size, the lack of players' characteristics as inputs (e.g., age and genre), and the non-probabilistic sampling method are the main factors that hampers the generalization of our findings, threatening our experiment external

\footnotetext{
${ }^{2}$ Available at: http: //twixar.me/f511 (in the file "best rules")
} 
VIII Congresso Brasileiro de Informática na Educação (CBIE 2019)

Anais do XXX Simpósio Brasileiro de Informática na Educação (SBIE 2019)

validity [Wohlin et al. 2012]. These limitations were remedied acknowledging that our findings are specific to our sample and that further research is yet necessary. Apart from those, there also are few threats to our study's internal validity because it involves subjects playing a game and then completing a questionnaire [Wohlin et al. 2012]. During the process, players could get tired, provided biased answers because they were aware to be participating in a research, beside being affected by external factors. Although those are hard to mitigate, player could stop playing the game, e.g. if they got tired, at any time. Also, the selected questionnaire is a validated, widely used instrument. In addition, the adopted version (in-game) was especially developed to situations as the one of this experiment. Lastly, despite aware of being participating in a research, players had privacy to complete the questionnaire without any pressure and could provide anonymous answers because their nickname did not have to reflect their real name. Nevertheless, there are threats to this study's validity, which were mostly remedied and, therefore, we argue to be insufficient to invalidate our experiment's findings.

\section{Concluding Remarks}

Based on the recent big challenge of providing automatic user students' experience in educational games, we proposed a data-driven based approach associating the student's' data $\operatorname{logs}$ and their game experience. We identified a different association between the data logs and the students' game experience that can be used to automatically identify the students game experience in real time. As a future study, we aim to implement an open source module to identify the real-time user experience in educational games, capable to be plugged in different kind of educational games.

\section{Acknowledgements}

The authors would like to thank the funding provided by FAPESP (Projects: 2018/076881; 2018/15917-0; 2016/02765-2; 2018/11180-3), CAPES and CNPq.

\section{References}

Bauckhage, C., Kersting, K., Sifa, R., Thurau, C., Drachen, A., and Canossa, A. (2012). How players lose interest in playing a game: An empirical study based on distributions of total playing times. In 2012 IEEE Conference on Computational Intelligence and Games (CIG), pages 139-146.

Bentler, P. M. and Chou, C.-P. (1987). Practical issues in structural modeling. Sociological Methods \& Research, 16(1):78-117.

Borges, S. S., Durelli, V. H., Reis, H. M., Bittencourt, I. I., Mizoguchi, R., and Isotani, S. (2017). Brazilian Portuguese Cross-Cultural Adaptation and Validation of the Susceptibility to Persuasion Scale (Br-STPS). In Proceedings - IEEE 17th International Conference on Advanced Learning Technologies, ICALT 2017, pages 72-73.

Charleer, S., Gerling, K., Gutiérrez, F., Cauwenbergh, H., Luycx, B., and Verbert, K. (2018). Real-time dashboards to support esports spectating. In Proceedings of the 2018 Annual Symposium on Computer-Human Interaction in Play, pages 59-71. ACM.

Dziuban, C. D. and Shirkey, E. C. (1974). When is a correlation matrix appropriate for factor analysis? some decision rules. Psychological bulletin, 81(6):358. 
VIII Congresso Brasileiro de Informática na Educação (CBIE 2019)

Anais do XXX Simpósio Brasileiro de Informática na Educação (SBIE 2019)

Eckardt, L. and Robra-Bissantz, S. (2018). Playtesting for a better gaming experience: Importance of an iterative design process for educational games research-in-progress. In Twenty-Second Pacific Asia Conference on Information Systems.

Hoaglin, D. C., Mosteller, F., and Tukey, J. W. (2000). Understanding robust and exploratory data analysis. Number Sirsi) i9780471384915.

Hooshyar, D., Yousefi, M., and Lim, H. (2017). A systematic review of data-driven approaches in player modeling of educational games. Artificial Intelligence Review, pages $1-21$.

Hooshyar, D., Yousefi, M., and Lim, H. (2018). Data-driven approaches to game player modeling: a systematic literature review. ACM Computing Surveys (CSUR), 50(6):90.

Huang, G.-B., Zhou, H., Ding, X., and Zhang, R. (2011). Extreme learning machine for regression and multiclass classification. IEEE Transactions on Systems, Man, and Cybernetics, Part B (Cybernetics), 42(2):513-529.

IJsselsteijn, W., De Kort, Y., and Poels, K. (2013). The game experience questionnaire. Eindhoven: Technische Universiteit Eindhoven.

Kaur, G. and Chhabra, A. (2014). Improved j48 classification algorithm for the prediction of diabetes. International Journal of Computer Applications, 98(22).

Kaur, H. (2018). A literature review from 2011 to 2014 on student's academic performance prediction and analysis using decision tree algorithm. Journal of Global Research in Computer Science, 9(5):10-15.

Lara, C. A., Mitre-Hernandez, H., Flores, J., and Perez, H. (2018). Induction of emotional states in educational video games through a fuzzy control system. IEEE Transactions on Affective Computing, pages 1-1.

Likert, R. (1932). A technique for the measurement of attitudes. Archives of psychology.

Loehlin, J. C. (1998). Latent variable models: An introduction to factor, path, and structural analysis. Lawrence Erlbaum Associates Publishers.

Mekler, E. D., Bopp, J. A., Tuch, A. N., and Opwis, K. (2014). A systematic review of quantitative studies on the enjoyment of digital entertainment games. In Proceedings of the 32nd annual ACM conference on Human factors in computing systems, pages 927-936. ACM.

Oliveira, W., Toda, A., Palomino, P., Rodrigues, L., Shi, L., and Isotani, S. (2019). Towards automatic flow experience identification in educational systems: A theorydriven approach. In Proceedings of the 2019 Annual Symposium on Computer-Human Interaction in Play Companion Extended Abstracts. ACM.

Pedersen, C., Togelius, J., and Yannakakis, G. N. (2010). Modeling player experience for content creation. IEEE Transactions on Computational Intelligence and AI in Games, 2(1):54-67.

Petri, G. and von Wangenheim, C. G. (2016). How to evaluate educational games: a systematic. Journal of Universal Computer Science, 22(7):992-1021. 
VIII Congresso Brasileiro de Informática na Educação (CBIE 2019)

Anais do XXX Simpósio Brasileiro de Informática na Educação (SBIE 2019)

Rodrigues, L., Bonidia, R. P., and Brancher, J. D. (2017). A math educacional computer game using procedural content generation. In Brazilian Symposium on Computers in Education, volume 28, page 756.

Rodrigues, L. and Brancher, J. D. (2019). Playing an educational game featuring procedural content generation: Which attributes impact players' curiosity? RENOTE, $17(1)$.

Rodrigues, L. A. L. and Brancher, J. D. (2018). Improving players' profiles clustering from game data through feature extraction. In 2018 17th Brazilian Symposium on Computer Games and Digital Entertainment (SBGames), pages 177-17709.

Santos, W. O., Gomes, T., and Silva, C. (2017). Towards to flow state identification in educational games: An empirical study. In Brazilian Symposium on Computers in Education, volume 28, page 927.

Santos, W. O. and Isotani, S. (2018). Desenvolvimento de jogos educativos? desafios, oportunidades e direcionamentos de pesquisa. RENOTE, 16(2).

Shaker, N., Yannakakis, G., and Togelius, J. (2010). Towards automatic personalized content generation for platform games. In Sixth Artificial Intelligence and Interactive Digital Entertainment Conference.

Spector, P. E. (1994). Using self-report questionnaires in ob research: A comment on the use of a controversial method. Journal of organizational behavior, 15(5):385-392.

Viera, A. J., Garrett, J. M., et al. (2005). Understanding interobserver agreement: the kappa statistic. Fam med, 37(5):360-363.

Wohlin, C., Runeson, P., Hst, M., Ohlsson, M. C., Regnell, B., and Wessln, A. (2012). Experimentation in Software Engineering. Springer Publishing Company, Incorporated.

Wood, R. D. T., Griffiths, M. K. D., and Eatough, V. A. (2004). Online data collection from video game players: Methodological issues. CYBERPSYCHOLOGY \& BEHAVIOR, 7(5):511-518.

Wu, X., Kumar, V., Quinlan, J. R., Ghosh, J., Yang, Q., Motoda, H., McLachlan, G. J., Ng, A., Liu, B., Philip, S. Y., et al. (2008). Top 10 algorithms in data mining. Knowledge and information systems, 14(1):1-37.

Xu, H., Wu, Z., Lin, T., Tang, N., and Hua, L. (2016). Automatic content generation in tetris game based on emotion modeling. In 2016 Nicograph International (NicoInt), pages $139-139$.

Yannakakis, G. N., Spronck, P., Loiacono, D., and André, E. (2013). Player Modeling. In Lucas, S. M., Mateas, M., Preuss, M., Spronck, P., and Togelius, J., editors, Artificial and Computational Intelligence in Games, volume 6 of Dagstuhl Follow-Ups, pages 45-59. Schloss Dagstuhl-Leibniz-Zentrum fuer Informatik, Dagstuhl, Germany.

Yannakakis, G. N. and Togelius, J. (2011). Experience-driven procedural content generation. IEEE Transactions on Affective Computing, 2(3):147-161.

Zammitto, V. (2009). Game research, measuring gaming preferences. In Proceedings of the 2009 conference on future play on@ GDC Canada, pages 15-16. ACM. 